\title{
Technical study for complete one-time sealing of vacuum interrupter
}

\author{
Congjun Xue*, Xiaozhao Li, Shibai Liu, and Fangshuai Zhao \\ Tianjin Pinggao Intelligent Electric Co. , Ltd. , 300300Tianjin, China
}

\begin{abstract}
This paper briefly describes the development process of sealing technology for vacuum interrupter, analyze the main characteristics of each stage and difficulties in realizing one-time sealing. Five design schemes are put forward to achieve complete one-time sealing, and the vacuum brazing tests were carried out according to the scheme. The test samples were dissected and analyzed, This article it contrastive analysis. The contact structure was chosen as the best structure for a complete one-time sealing, and made into a vacuum interrupter. The vacuum interrupter has passed the vacuum, the contact resistance, the power-frequency voltagewithstand test, BIL and the breaking test. It is proved that the one-time sealing of this structure can meet the requirements.
\end{abstract}

\section{Introduction}

With the continuous improvement of vacuum switch manufacturing technology and theoretical research level, vacuum interrupter has become a leading product in the medium voltage field by virtue of its huge technical advantages. Vacuum interrupters have been continuously developing in the direction of miniaturization, high reliability and low cost. At the same time, it continues to penetrate into the low voltage and high voltage fields. On the basis of ensuring quality, enterprises continuously improve the manufacturing technology of products and reduce the manufacturing cost, which is the necessary means to open up the market and expand users. The transformation of manufacturing technology includes new technologies, new structures and new materials. Manufacturers have increased production efficiency and reduced production costs by improving the process level (such as the popularization of one-time sealing process).

\section{Sealing process of vacuum interrupter}

The development of vacuum interrupter sealing and exhausting processes has experienced a long history from the 1960 s to today. Mainly can be summarized as three major stages: baking exhaust of exhaust platform ${ }^{[1-2]}$, one-time sealing of vacuum furnace; complete onetime sealing of vacuum furnace ${ }^{[3-5]}$.

In the first stage, from the 1960 s to the early 1980 s, the sealing and exhaust process of the vacuum interrupter was completely separated. It mainly consists of five parts: First, the

* Corresponding author: xuecjpg@126.com 
welding of the contact piece and the conductive rod; Second, the welding of the shell portion of the vacuum interrupter; Third, the assembly and welding of the whole tube; Fourth, baking and exhaust on the exhaust platform; Fifth, the whole tube is separated from the exhaust. This technical process is long, and each process is in a separate workplace, and the transportation and turnover of materials are troublesome. In this turnover process, it is possible to cause surface contamination or bumps in parts. It will have an impact on the final quality of the vacuum interrupter.

In the second stage, it is sealed once in a vacuum furnace. This technology is a relatively mature technology that is currently widely used. The process is to braze the contact sheet and conductive rod in vacuum furnace. Then, all the parts are assembled again and placed in a vacuum furnace for final exhausting and welding. The production process of the entire vacuum interrupter is shortened, and the assembly and welding of the vacuum interrupter can be carried out in a clean room. This has made a qualitative leap in the manufacturing quality of vacuum interrupters.

In the third stage, it is a complete one-time sealing process. This technique requires all parts to be assembled and placed in a vacuum furnace at a time after the final cleaning parts into the clean room, and the exhausting and welding processes are completed at one time. This technology has the shortest process. Regardless of the quality of the vacuum interrupter or the cost of production, this technology is currently the most suitable sealing process for vacuum interrupters.

The sealing and brazing process of the vacuum interrupter plays a very important role in maintaining the vacuum degree of the vacuum interrupter. The process route with complete one-time sealing is short, the assembly efficiency is high, and the primary yield is high, which is suitable for large-scale production; the process does not need to carry out parts welding, the manual link is the least, the process is simple and easy to ensure the stability of product quality, product consistency it is good. Therefore, it is necessary to study the complete one-time sealing technology to ensure the stability of product quality. However, there is a quality hazard in the welding of the conductive rod and the contact piece, which needs to be improved from the technical process.

\section{Test method}

The premise of achieving a complete one-time sealing is to complete the welding of the components in one-time sealing process in the whole tube sealing. When vacuum brazing, the moving parts of vacuum interrupter is suspended in the vacuum interrupter, Therefore, it is necessary to rationally select the moving conductive rod and the contact piece, and solve the welding problem of the moving parts to realize the complete one-time sealing process.

In this research scheme, the transverse magnetic field contact structure is used, such as Figure 3 (a), and five kinds of welded structures are proposed. Each structure is suspended and welded, and the welded structure is dissected, and the weld condition is analyzed. The five structures are comparatively analyzed to determine that it is suitable for a complete one-time sealing, as Figure 3.
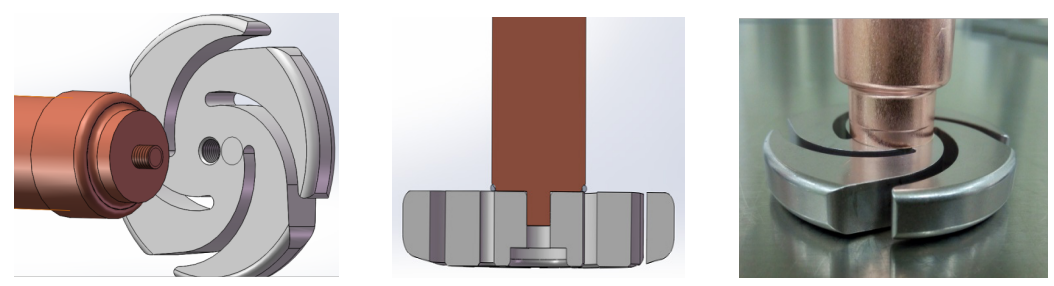
(a)

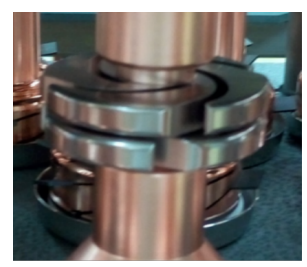

(d) (b)

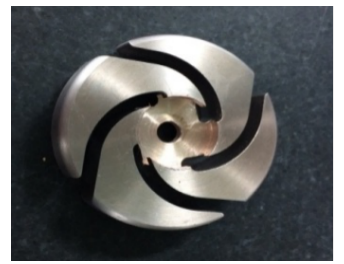

(e) (c)

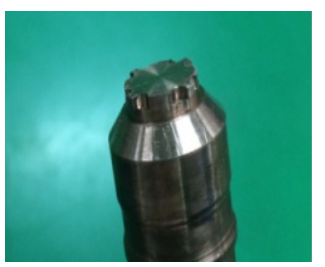

(f)

Fig. 3. Welding method.

1)Riveting type: The contact piece and the conductive rod are fixed together by a riveting press, and solder is added at the joint.

2)Thread type: The conductive rod and the contact piece are threaded together, the welding surface of the conductive rod is positioned to protrude the external thread, the middle hole of the contact piece is machined with the internal thread, and the contact surface is added with the solder ring.

3)Tight fit type: The conductive rod and the contact piece are designed to be interference fit welding.

4)Contact type: The welding is carried out by means of contact welding of the moving contact piece and the static contact piece, and the static contact piece is used as the platform for welding the moving conductive rod and the moving contact piece.

5) Card slot type: The moving conductive rod and the contact piece are fixed by the card slot, and the solder is added to the joint to enter the vacuum furnace for welding.

Five samples were prepared for each welding method. After the welding was completed, the samples were dissected and ground, and the contact surface of the conductive rod and the contact piece was observed by a metallographic microscope, as shown in Figure 4, and the welding condition was analyzed.

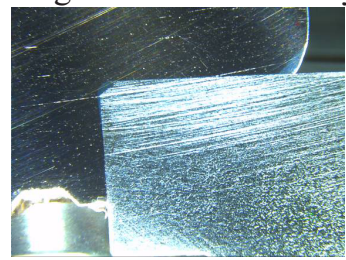

(a)

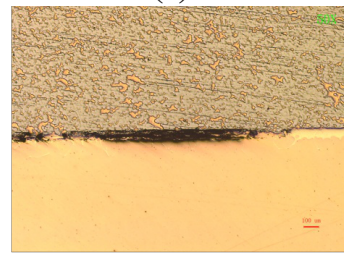

(d)

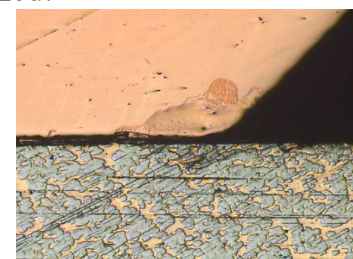

(b)

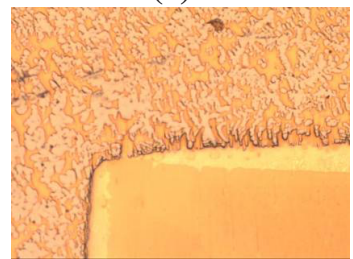

(e)

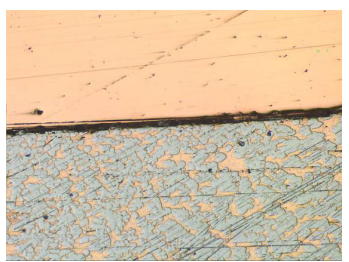

(c)

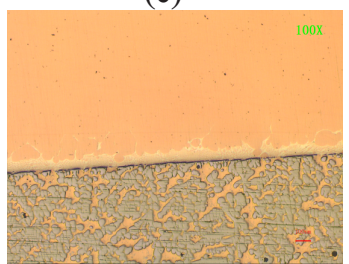

(f)

Fig. 4. State after welding.

Table 1. Welding statistics.

\begin{tabular}{|c|c|c|c|}
\hline Structure type & $\begin{array}{c}\text { Quantity of } \\
\text { samples }\end{array}$ & $\begin{array}{c}\text { Qualified } \\
\text { Quantity }\end{array}$ & $\begin{array}{c}\text { Qualification } \\
\text { rate }\end{array}$ \\
\hline Riveting type & 5 & 2 & $40 \%$ \\
\hline Thread type & 5 & 0 & 0 \\
\hline Tight fit type & 5 & 1 & $20 \%$ \\
\hline
\end{tabular}




\begin{tabular}{|c|c|c|c|}
\hline Contact type & 5 & 5 & $100 \%$ \\
\hline Card slot type & 5 & 5 & $100 \%$ \\
\hline
\end{tabular}

As shown in Figure 4 and Table 1, there are weld defects in riveting type, screw type and interference fit type. The solder cannot fill the weld join and cannot be used as a complete one-time sealing structure. There is no weld defect in all the 5 samples of contact type and card slot type, which can be used as a complete one-time sealing structure of vacuum interrupter. After the test, the conclusion is as shown in Table 2.

Table 2. Advantages and disadvantages of the structure.

\begin{tabular}{|c|c|c|}
\hline Structure type & Advantages & Disadvantages \\
\hline Riveting type & Easy to process and assemble & $\begin{array}{c}\text { Unreliable welding, there are } \\
\text { welding hazards }\end{array}$ \\
\hline Thread type & Easy to process and assemble & $\begin{array}{c}\text { Unreliable welding, there are } \\
\text { welding hazards }\end{array}$ \\
\hline Tight fit type & Easy to process and assemble & $\begin{array}{c}\text { The conductive rod needs to be } \\
\text { pickled, the interference size } \\
\text { cannot be guaranteed, and the } \\
\text { welding is not reliable. }\end{array}$ \\
\hline Contact type & Easy to process and weld firmly & Assembly is difficult \\
\hline Card slot type & Simple assembly and firm welding & Difficulties in processing parts \\
\hline
\end{tabular}

Through the above comparison and analysis, considering the welding situation and the difficulty of processing and assembly, 5 Vacuum Interrupters were welded by contact type They were tested, the vacuum degree $\geqslant 1 \mathrm{E}-4 \mathrm{pa}$, the resistance $\leqslant 8 \mu \Omega$, Rated powerfrequency withstand voltage is $48 \mathrm{kV}$, Rated lightning impulse withstand voltage is $85 \mathrm{kV}$, and the breaking test was carried out. The number of breaking times of each vacuum interrupter is 30 times. Through these tests, it is proved that a complete one-time sealing of the contact type is feasible.

\section{Conclusion}

Through the process research of five kinds of welded structures, the welded structure was dissected and analyzed. The welded structure was dissected and analyzed, and the contact type was selected for complete one-time sealing to weld vacuum interrupter. The appearance welds and the internal weld of the vacuum interrupter were good. The vacuum interrupter passed the factory test and was successfully interrupted 30 times. The contact welding method achieves a complete one-time sealing process, eliminating the separate welding of the contact piece and the conductive rod. The vacuum interrupter of contact welding was dissected and analyzed, and compared with the vacuum interrupter produced by one-time sealing, the solder melts and fills the weld. The solder melts and fills the weld, which can meet the requirements.

\section{References}

1. Yao Shibin. HIGH VOLTAGE APPARATUS, 46-48(1999)

2. Gu Shuyi. Vacuum Electronics, 56-59(1992)

3. Wang Zheng, Fei Guangcheng, Liu Ying. HIGH VOLTAGE APPARATUS, 6364,67(2003)

4. Zhang Yujie, Zhao Lihua. low voltage apparatus, 51-53(1999)

5. Pan qian. Vacuum Electronics, 57-59(1999) 